\title{
Myasthenia Gravis and Its Comorbidities
}

\author{
Bernardo Cacho Diaz ${ }^{1^{*}}$, Paola Flores-Gavilán², Guillermo García-Ramos ${ }^{3}$ and Nydia A. Lorenzana-Mendoza ${ }^{2}$ \\ ${ }^{1 *}$ Neurology coordinator at the Instituto Nacional de Cancerología (National Cancer Institute), Mexico City. Av. San Fernando 22. Col. Sección XVI. ZC 14,080. Mexico. \\ ${ }^{2}$ Universidad Nacional Autónoma de México \\ ${ }^{3}$ Guillermo Garcia-Ramos MD, Head of Neurology Department. Instituto Nacional de Ciencias Médicas Salvador Zubiran, Mexico City \\ *Corresponding author: Bernardo Cacho Diaz, Neurology coordinator at the Instituto Nacional de Cancerología (National Cancer Institute), Mexico City. Av. San \\ Fernando 22. Col. Sección XVI. ZC 14,080. Mexico City, Tel: (525) 2127-1049; E-mail: bernardocacho@doctor.com
}

Received date: September 16, 2015; Accepted date: September 22, 2015; Published date: September 29,2015

Copyright: ( 2015 Bernardo CD, et al. This is an open-access article distributed under the terms of the Creative Commons Attribution License, which permits unrestricted use, distribution, and reproduction in any medium, provided the original author and source are credited.

\begin{abstract}
Background: Myasthenia gravis (MG) is an unpredicted neurologic disorder that may cause death; previous reports have failed to find a prognostic marker for the disease.

Aim: To determine the impact of comorbidities in patients with Myasthenia gravis.

Material and Methods: From January of 2002 to February of 2008 a database was created for patients with Myasthenia gravis (MG). The following variables were studied: age at onset of MG, gender, diabetes mellitus, dyslipidemia, arterial hypertension, dysthyroidism, autoimmune disease, thymectomy procedure and histopathologic result, myasthenic crisis, emergency room visits due to weakness, use and maximum dose of pyridostigmine, prednisone and azathioprine.
\end{abstract}

Results: In a total of 253 patients, we found comorbidities in $73 \%$. The most frequent associated disorders were dyslipidemia, thyroid disease, diabetes, hypertension and other autoimmune conditions. Patients with MG and thymoma, diabetes, dyslipidemia, dysthyroidism and/or hypertension had higher rates of ER visits, myasthenic crises, and required higher doses of drugs.

Conclusions: This study demonstrates that comorbidities are frequent in patients with $\mathrm{MG}(73 \%)$ and that they might worsen the prognosis of MG.

\section{Keywords: Myasthenia Gravis, Comorbidity, Thymoma, Autoimmune, Prognosis. \\ Introduction \\ Myasthenia gravis (MG) is an autoimmune disease characterized by fluctuating weakness of striated muscles, which can be severe enough to cause death. Several reports describe comorbidities in patients with myasthenia gravis [1-6], but most of these report autoimmune rather than metabolic diseases. The relationship between comorbidities and prognosis of MG has not yet been elucidated. Both incidence and prevalence of MG and other diseases vary according to the region of the world under study [7-11].}

A history of fluctuating weakness of voluntary muscles and a detailed neurological exam are the most useful tools in diagnosing MG. It is important to confirm the clinical diagnosis with at least one lab test such as the anti-ACh receptor antibodies determination, single fibre electromyography, repetitive electrostimulation (Jolly test), ice or edrophonium test [12]. Every patient with MG should get a chest CT to rule out the presence of thymoma. Other diseases must be ruled out before confirming the diagnosis [13].

Treatment for MG includes a limited gamut of interventions and there is not a standardized scheme, so each case should be individualized according to stated recommendations [14].
Myasthenic crisis (MC) is defined as an acute exacerbation of myasthenic weakness leading to ventilatory failure often requiring mechanical support. Approximately 30\% of all patients with MG develop ventilatory weakness and at least 15 to $20 \%$ suffer myasthenic crises (MC). Of patients who survive a first crisis, one third experience a second [15-16].

\section{Material and Methods}

The objective of this study was to determine the impact of comorbidities in patients with MG in a mestizo Hispanic population.

All patients included in this study had MG diagnosed clinically and on the basis of at least two confirmatory tests. Both main investigators (BC, GGR) met them at least once. They were seen for neurology consult at a referral center from 2002 to 2008 .

The following data was obtained: age at the onset of MG, gender, results of the following tests: repetitive electrostimulation and/or single fibre electromyography, edrophonium, anti-acetylcholine receptor antibodies (Ach-R Ab), presence of ocular or systemic symptoms, thyroid function tests (TSH, T3, T4, CT3, ITL), anti-thyroid antibodies, number of visits to emergency room (ER) caused by weakness, number of myasthenic crises, maximum dose and use of pyridostigmine, prednisone and/or azathioprine. Information was obtained about whether the patients had thymectomy or not, the histopathology, and the presence or absence of comorbidities such as 
Citation: Bernardo Cacho Diaz, Paola Flores-Gavilán, Guillermo García-Ramos and Nydia A. Lorenzana-Mendoza (2015) Myasthenia Gravis and Its Comorbidities. J Neurol Neurophysiol 6: 317. doi:10.4172/2155-9562.1000317

Page 2 of 5

diabetes mellitus, arterial hypertension, dyslipidemia, thyroid disease or any other disease.

Blood samples were obtained from patients when they were not hospitalized, had no acute disease (i.e. infection) and were not receiving high doses of steroids $(>0.5 \mathrm{mg} / \mathrm{kg} / \mathrm{d}$ of prednisone or its equivalent).

Exclusion criteria: unsupported MG diagnosis or a different established diagnosis, incomplete file or medical follow-up of less than 12 months.

\section{Statistical analysis}

Clinical and pathological manifestations in patients with MG with or without comorbidities were compared with Student T and MannWhitney $\mathrm{U}$ tests for continuous variables with or without normal distribution, respectively; ANOVA and Turkey correction were used to analyze $>3$ variables, odds ratio was used to compare prognostic observations.

\section{Ethical aspects} IRB.

The study did not raise ethical concerns and was approved by the

\section{Results}

A total of 253 patients were included: 178 were women (70\%) and 75 men (30\%), with a female-to-male ratio of 2.4:1. The average age of onset of symptoms was $35 \pm 17$ years [31 \pm 15 for women and $44 \pm 18$ for men $(\mathrm{P}<0.001)]$. Information about the distribution and severity of weakness was obtained in 252 patients. Sixteen patients (7\%) had purely ocular and 236 (93\%) generalized weakness. Most patients (79\%, n 200) developed symptoms before 50 years of age. Repetitive electrostimulation results (Jolly's test) were obtained in 236 patients, being positive in 219 (93\%) with an average electrodecrement of 32.5\% (Range: 11-118). Acetylcholine anti-receptor antibodies (AchR-ab) results were obtained in 228 patients, being positive in 199 (87\%). Ninety percent of patients with systemic manifestations and $50 \%$ with purely ocular had positive AChR ( $\mathrm{p}<0.001)$. The edrophonium test was performed in 144 patients and was reported as positive in 137 (95\%).

Of the studied patients, 84 (33\%) had myasthenic crisis (MC) and $150(60 \%)$ sought assistance due to weakness. In total, $73 \%$ of our patients presented comorbidities. The prognosis according to each comorbid condition is summarized in Table 1 .

\begin{tabular}{|c|c|c|c|c|c|c|c|c|c|c|c|}
\hline & \multirow{2}{*}{$\begin{array}{l}\text { Age }(y) \pm \\
\text { SD }\end{array}$} & \multirow{2}{*}{ Fem/Mae } & \multirow{2}{*}{ MC n (\%) } & \multirow{2}{*}{$\begin{array}{l}\text { No. Crisis } \pm \\
\text { SD }\end{array}$} & \multirow{2}{*}{ ER n (\%) } & \multirow{2}{*}{$\begin{array}{l}\text { No. ER } \pm \\
\text { SD }\end{array}$} & PDM dose & \multirow{2}{*}{$\begin{array}{l}\text { PDN use } \mathrm{n} \\
(\%)\end{array}$} & \multirow{2}{*}{$\begin{array}{l}\text { PDN } \\
\text { dose mg } \\
\pm \text { SD }\end{array}$} & \multirow{2}{*}{$\begin{array}{l}\text { AZA use } \\
\mathrm{n}(\%)\end{array}$} & \multirow{2}{*}{$\begin{array}{l}\text { AZA dose } \\
\mathrm{mg} \pm \mathrm{SD}\end{array}$} \\
\hline & & & & & & & $\mathrm{mg} \pm \mathrm{SD}$ & & & & \\
\hline \multicolumn{12}{|c|}{ Comorbidity (n 252) } \\
\hline $\begin{array}{ll}\begin{array}{l}\text { Yes } \\
(73 \%)\end{array} & 185 \\
(73)\end{array}$ & $38 \pm 18$ & $63 / 37$ & $64(35)$ & $1.8 \pm 1.2$ & $118(64)$ & $3.3 \pm 3$ & $360 \pm 180$ & $139(75)$ & $49 \pm 26$ & $87(47)$ & $77 \pm 26$ \\
\hline No & $25 \pm 9$ & $91 / 9$ & $20(30)$ & $1.3 \pm 0.7$ & $32(48)$ & $2 \pm 2$ & $328 \pm 139$ & $38(57)$ & $37 \pm 18$ & $19(28)$ & $68 \pm 26$ \\
\hline$P$ & $<0.0001$ & $<0.0001$ & NS & NS & 0.016 & 0.005 & NS & 0.004 & 0.011 & 0.006 & NS \\
\hline OR 95\% Cl & & & $1.2(0.7-2.3)$ & & $1.92(1.1-3.4)$ & & & & & & \\
\hline \multicolumn{12}{|c|}{ Dyslipidemia (n 234) } \\
\hline $\begin{array}{ll}\text { Yes } & 140 \\
(60 \%) & \end{array}$ & $39 \pm 18$ & $63 / 37$ & $50(36)$ & $1.7 \pm 1.2$ & $94(67)$ & $3.4 \pm 3.1$ & $373 \pm 182$ & $106(76)$ & $49 \pm 27$ & $70(50)$ & $79 \pm 27$ \\
\hline No & $31 \pm 16$ & $80 / 20$ & $27(29)$ & $1.7 \pm 1.2$ & $45(48)$ & $2.8 \pm 2.8$ & $321 \pm 148$ & $60(64)$ & $42 \pm 21$ & $32(34)$ & $66 \pm 24$ \\
\hline$P$ & 0.001 & 0.004 & NS & NS & 0.003 & NS & 0.023 & 0.035 & NS & 0.011 & 0.015 \\
\hline OR 95\% Cl & & & $1.4(0.8-2.4)$ & & $2.2(1.3-3.8)$ & & & & & & \\
\hline \multicolumn{12}{|c|}{ Dysthyroidism (n 233) } \\
\hline Yes $45(19 \%)$ & $37 \pm 17$ & $76 / 24$ & $15(33)$ & $2 \pm 1.4$ & $35(78)$ & $3.8 \pm 3.1$ & $374 \pm 204$ & $33(73)$ & $49 \pm 31$ & $20(44)$ & $73 \pm 21$ \\
\hline No & $34 \pm 17$ & $70 / 30$ & $61(32)$ & $1.6 \pm 1$ & 109 (58) & $2.8 \pm 2.8$ & $348 \pm 162$ & $132(70)$ & $46 \pm 24$ & $82(44)$ & $76 \pm 28$ \\
\hline$P$ & NS & NS & NS & NS & 0.01 & NS & NS & NS & NS & NS & NS \\
\hline OR 95\% Cl & & & $1.0(0.5-2.1)$ & & $2.5(1.2-5.4)$ & & & & & & \\
\hline \multicolumn{12}{|c|}{ Diabetes mellitus ( $n$ 252) } \\
\hline Yes $50(20 \%)$ & $46 \pm 17$ & $56 / 44$ & $24(48)$ & $1.7 \pm 0.9$ & $40(80)$ & $3.6 \pm 3.6$ & $410 \pm 204$ & 34 (68) & $52 \pm 21$ & 27 (54) & $82 \pm 28$ \\
\hline No & $32 \pm 16$ & $74 / 26$ & $60(30)$ & $1.7 \pm 1.2$ & $110(55)$ & $2.8 \pm 2.6$ & $337 \pm 158$ & $143(71)$ & $45 \pm 26$ & 79 (39) & $73 \pm 25$ \\
\hline $\mathrm{P}$ & $<0.0001$ & 0.012 & 0.012 & NS & 0.001 & NS & 0.006 & NS & 0.029 & 0.041 & NS \\
\hline
\end{tabular}




\begin{tabular}{|c|c|c|c|c|c|c|c|c|c|c|c|}
\hline OR 95\% Cl & & & $2.2(1.2-4.1)$ & & $3.4(1.6-7.1)$ & & & & & & \\
\hline \multicolumn{12}{|c|}{ Hypertension (n 252) } \\
\hline Yes $39(15 \%)$ & $54 \pm 15$ & $51 / 49$ & $17(44)$ & $1.9 \pm 1.2$ & $31(80)$ & $3.4 \pm 2.7$ & $366 \pm 203$ & $33(85)$ & $48 \pm 18$ & $22(56)$ & $81 \pm 31$ \\
\hline No & $31 \pm 15$ & $74 / 26$ & $67(31)$ & $1.6 \pm 1.1$ & $119(56)$ & $2.9 \pm 2.9$ & $349 \pm 164$ & $144(68)$ & $46 \pm 26$ & $84(39)$ & $74 \pm 25$ \\
\hline$P$ & $<0.0001$ & 0.005 & NS & NS & 0.004 & NS & NS & 0.022 & NS & 0.037 & NS \\
\hline OR 95\% Cl & & & $1.7(0.8-3.4)$ & & $3.1(1.4-7)$ & & & & & & \\
\hline \multicolumn{12}{|c|}{$\begin{array}{l}\text { Autoinmune desease ( } \\
\text { 253) }\end{array}$} \\
\hline Yes $17(7 \%)$ & $30 \pm 12$ & $94 / 6$ & $2(12)$ & $1 \pm 0$ & $7(41)$ & $2.4 \pm 2.6$ & $367 \pm 219$ & $13(77)$ & $43 \pm 14$ & $6(36)$ & $92 \pm 34$ \\
\hline No & $35 \pm 17$ & $69 / 31$ & $82(35)$ & $1.7 \pm 1.1$ & $143(61)$ & $3.1 \pm 2.9$ & $351 \pm 167$ & $164(70)$ & $46 \pm 26$ & $100(43)$ & $74 \pm 26$ \\
\hline$P$ & NS & 0.018 & 0.039 & NS & NS & NS & NS & NS & NS & NS & NS \\
\hline OR 95\% Cl & & & $0.3(0.1-1.1)$ & & $0.5(0.2-1.2)$ & & & & & & \\
\hline \multicolumn{12}{|c|}{ Thymoma (n 199) } \\
\hline Yes $30(15 \%)$ & $44.5 \pm 14$ & $30 / 70$ & $16(53)$ & $1.6 \pm 0.7$ & $21(70)$ & $2.9 \pm 2.3$ & $405 \pm 253$ & $27(90)$ & $48 \pm 27$ & $17(57)$ & $72 \pm 23$ \\
\hline No & $29.5 \pm 13$ & $82 / 18$ & $50(30)$ & $1.8 \pm 1.3$ & $95(56)$ & $3.3 \pm 3.3$ & $368 \pm 154$ & $115(68)$ & $46 \pm 27$ & $64(38)$ & $78 \pm 28$ \\
\hline$P$ & $<0.0001$ & $<0.0001$ & 0.011 & NS & NS & NS & NS & 0.009 & NS & 0.043 & NS \\
\hline OR 95\% Cl & & & $2.7(1.2-6)$ & & $1.8(0.8-4.2)$ & & & & & & \\
\hline
\end{tabular}

Table 1: Prognosis of patients with Myasthenia gravis according to the presence or absence of comorbidities: Dyslipidemia, Thyroid disease, Diabetes, Hypertension, Autoimmune disease, Thymoma. AZA = Azathioprine, SD = Standard deviation, ER = Emergency Room visits, Fem= Female, Masc $=$ Male, $\mathrm{MC}=$ Myasthenic crisis, No. = number, NS= Non significant, $\mathrm{PDM}=$ pyridostigmine, $\mathrm{PDN}=$ prednisone, $\mathrm{OR}=\mathrm{Odds}$ ratio, $\mathrm{CI}=$ confidence interval

Almost 200 patients were submitted to thymectomy and the histopathology of the thymus was available in 199, 30 (15\%) were positive for thymoma (MGT). Of the MGT group (n 30), the onset of MG was between $30-70$ years, 16 (53\%) had at least one MC, $70 \%$ required ER services; for the 169 patients without thymoma (MGNT), the age at onset ranged from 10-40 years, 50 (30\%) had MC and 56\% required ER services. MGT patients showed a higher frequency of autoimmune diseases (10 vs $7 \%$ ).

Thyroid function test results were obtained in 233 patients and alterations were found in 45 (19\%), indicating hyperthyroidism in 14 (31\%) and hypothyroidism in 31 (69\%). In the overall cohort, $13 \%$ presented hypothyroidism and $6 \%$ presented hyperthyroidism; of the patients with dysthyroidism, $98 \%$ presented a generalized form of MG and only one case presented with a pure ocular form, of the 15 patients with purely ocular symptoms, only one presented dysthyroidism. A lipid profile was obtained in 234 patients and dyslipidemia was diagnosed in $60 \%$. Glucose levels or glucose tolerance tests were performed in 252 patients and diabetes mellitus (DM) was diagnosed in $20 \%$. Blood pressure levels were determined at least twice in 253 patients; hypertension $(\mathrm{AH})$ was diagnosed in $16 \%$. All patients were questioned and examined to identify concomitant autoimmune diseases $(\mathrm{AD}) ; 21$ ADs were found in 17 patients, including 6 with rheumatoid arthritis, 3 autoimmune thrombocytopenic purpura, 2 systemic lupus erythematosus, 2 anti-phospholipid syndrome, 2 vitiligo, 2 neuromyelitis optica, 1 uveitis, 1 pemphigus foliaceus, 1 cyclic autoimmune neutropenia, and 1 with Sjögren's syndrome.
In 6 patients we found 7 cases of non-thymic neoplasia, breast cancer being the most frequent $(n=3)$; followed by non-Hodgkin lymphoma, prostatic, thyroid or bladder cancer (one case each). Other diseases found in our patients were: stroke (2), hyperparathyroidism (2), interventricular communication, asthma, acromegaly, congenital lymphangioma and hyperprolactinemia (one case each).

\section{Discussion}

All patients included in the present study (n 253) had a definite diagnosis of MG. Few reports and series mention how their diagnoses were made; for this reason, in this study, we decided that every patient should fulfil well-defined and accepted diagnostic criteria [12] with the purpose of having solid results.

The average age of onset and the relation women: men of MG in this study $(7: 3)$ are similar to previous studies [17]. In regard to the affection form, $93 \%$ of the patients manifested a generalized presentation and only $7 \%$ a pure ocular form of the disease; this could be explained by a reference systematic error. However, other authors have found a similar proportion in the affection degree (Grob [17] et al $86 \%$ vs $14 \%$ and Kuks [7] et al $90 \%$ vs $10 \%$ ).

The AchR-Abs were positive in $87 \%$ of all patients and were more frequently positive in those with generalized (90\%) than in purely ocular presentations (50\%). 


\section{Thymoma}

Of patients submitted to thymectomy, $15 \%$ had thymoma, for a total of 30 cases (12\%) of the whole cohort. The frequency of MC was higher in our studied population than in earlier reports (33 vs $15-20 \%$ ), regardless of thymic pathology [15]. The MGT group had a later age at onset (45 \pm 14 years) than those without thymoma (MGNT) $(30 \pm 13$ y) $(p<0.0001)$, similar to previous reports [7]. The presence of thymoma seems to be an important risk factor for MC; in the present study $53 \%$ of MGT had MC OR of 2.7; 95\% CI, 1.2-6; others [7] also have associated a higher incidence of $\mathrm{MC}$ and a more aggressive disease in patients with thymoma.

\section{Co-morbidities}

Co-morbidity (concomitant disease besides MG) was found in 185 patients (73\%), the most common associations being: dyslipidemia 60\% (MGD), diabetes mellitus 20\% (MGDM), dysthyroidism 19\% (MGTD), hypertension 16\% (MGAH) and autoimmune diseases $7 \%$ (MGAD). Having a comorbid disease was associated more frequently with MC (OR 1.2) and ER visits (OR 1.92).

\section{Thyroid function and steroids in Myasthenia gravis}

Thyroid hormones have an influence on neuromuscular junction (NMJ); alterations have been demonstrated in $79 \%$ of hypothyroid and $67 \%$ of hyperthyroid patients with clinical weakness in $38 \%$ and $62 \%$ respectively [18]. These clinical manifestations significantly improve after thyroid function correction. The diagnosis of thyroid disease (TD) must be carefully considered and under certain conditions, not all alterations in thyroid function tests imply a diagnosis of TD [19].

TD is one of the most frequent comorbidities associated with MG, with a prevalence varying from $5 \%$ to $10 \%$, and MG is present in $0.2 \%$ of the patients diagnosed with autoimmune thyroid disease [4].

Acute disease and the use of steroids affect thyroid function, but steroids have a beneficial effect on MG and are used as treatment [20] due to its immunoregulator function and associated proliferation of Ach receptors [21].

Thyroid function tests were abnormal in $19 \%$ of the 233 studied patients; hypothyroidism was more frequent (69\%) than hyperthyroidism (31\%), different from previous data [17]. The reason for this high occurrence of thyroid alterations is unknown, but a systemic reference bias may play an important role. The diagnosis of TD was meticulous, making sure they were not hospitalized; on a high steroid dose or in acute conditions at the time the samples were taken. Patients studied showed higher frequency of TD than the general population [22] 19 vs. $5 \%$, meaning it is five times more common to find TD in patients with MG.

Patients with TD did not show worse prognosis overall, however, those without TD sought less attention due to weakness $78 \mathrm{vs} 58 \%$ OR of 2.5 ; $95 \% \mathrm{CI}, 1.2-5.4$. A pure ocular form was found in only $2 \%$ of the TD group vs $7 \%$ without TD, unlike previously reported [4]. Further investigations must be made to answer the role of TD on the prognosis of MG.

\section{Diabetes mellitus}

The association of Diabetes Mellitus (DM) and MG was first described in 1950 by Perry [23]. Since then, several authors have confirmed their coexistence [24-25]. The ADA (American Diabetes
Association) has established criteria for the diagnosis and treatment [26]. DM was identified in $20 \%$ of our patients, three times higher than in the general population (7\%) [27]. Patients with MGDM had later age of onset, than those without DM. Men were more likely to develop DM than women (29 vs $16 \%$, p 0.012). Patients with MGDM: a) had higher frequency of MC (48 vs $29.7 \%$, OR of 2.2 ; $95 \%$ CI, $1.2-41, b$ ) required more attention from the ER services ( 80 vs $54.5 \%$ ) OR of 3.4; 95\% CI 1.6-7.1, c) required higher mean dose of PDM (410 vs $337 \mathrm{mg} /$ day, p 0.006), d) higher mean dose of prednisone ( $52 \mathrm{vs} 45 \mathrm{mg} /$ day, $\mathrm{p}$ 0.029 ), and e) were prescribed with azathioprine more often (54 vs $39 \%$ ) (p 0.041); than those without DM. No differences in frequency of DM were found between patients using steroids or not; however, this relation cannot be dismissed by the present study.

\section{Arterial Hypertension}

This disease is the main risk factor for mortality in the world. Fifteen percent of our patients with MG were diagnosed with $\mathrm{AH}$ (MGAH); the prevalence of $\mathrm{AH}$ in patients with $\mathrm{MG}$ was half lower than in the general population (15 vs 30\%) [28]. Mean age of onset of MG symptoms was higher for patients with HT (54 vs 31 years) $(\mathrm{p}<0.0001)$. Males presented AH more frequently ( 25 vs $11 \%)$. Patients with $\mathrm{MG}$ and $\mathrm{AH}$ required more services from the ER than patients without $\mathrm{AH}$ ( 80 vs $56 \%$, OR 3.1 ) and had more MC (44 vs $31 \%$, OR 1.7). The use of steroids was higher in patients with MGAH (85 vs. $68 \%$ ) (p 0.022). Whether or not, the use of steroids is related to the presence of $\mathrm{AH}$ is a conclusion that this study cannot presume to reach.

\section{Dyslipidemia}

Dyslipidemia is one of the main risk factors for the development of cardiovascular diseases. The impact of this disorder in patients with MG has not yet been studied; however, it is known that treatment with statins is associated with the development or exacerbation of MG [29-31].

Dyslipidemia was diagnosed in $60 \%$ of the patients (MGD), more frequent in men ( $73 \%$ vs $54 \%)$ (p 0.004). Patients with MGD needed more ER services (68 vs 32\%) OR od 2.2; 95\% CI, 1.3-3.8 and had more MC (36 vs 29\%) OR 1.4, used higher mean dose of PDM (373 vs 321 $\mathrm{mg} / \mathrm{d}$ ) and were more often prescribed azathioprine (69 vs $31 \%$ ). This study does not discard that steroids are a risk factor for the presence of dyslipidemia; however, the occurrence of dyslipidemia in those without steroid treatment seems to be an independent factor associated with MG.

Mean age of symptom onset was greater in MGD (39 vs 31 years) (p 0.001 ). Higher prevalence of dyslipidemia was found for patients with MG than in the general population (60 vs $27 \%$ ) [28], even for patients that were not receiving steroids (50\%).

\section{Other autoimmune diseases}

Seventeen patients (7\%) presented a non-thyroid related autoimmune disease (MGAD). Women presented higher frequency of $\mathrm{AD}(9 \%$ vs $1 \%$ ), p 0.018 . MGAD patients had less MC ( 12 vs $35 \%$, OR 0.3 ) and required less ER visits ( 41 vs $61 \%$, OR 0.5 ); the etiology of this finding cannot be explained by the present study; further investigations should be performed to answer the association. No other differences were found between MGAD vs MG without AD. Our MG population had a similar prevalence of $\mathrm{AD}$ to those reported in other studies [7]. 


\section{Conclusions}

The present study demonstrates the elevated occurrence of comorbidities $(73 \%)$ in patients with $\mathrm{MG}$. The presence of dysthyroidism, dyslipidemia and diabetes mellitus was more frequent in patients with MG than in the general population, and the prevalence of $\mathrm{AH}$ was slightly lower.

Patients with MG should be screened for thyroid function, diabetes, hypertension, dyslipidemia, autoimmune diseases and thymoma. We found a $15 \%$ frequency of MGT in our patients. The frequency of other neoplastic disorders (non thymoma) seems to be increased in patients with MG.

According to the variables studied, we could assume that the prognosis of the MG worsens in the presence of thymoma, thyroid disease, DM, dyslipidemia and/or AH. This is the first investigation analysing concomitant metabolic diseases as prognostic factors in patients with MG.

\section{References}

1. Gotkine M, Fellig Y, Abramsky O (2006) Occurrence of CNS demyelinating disease in patients with myasthenia gravis. Neurology 67: 881-883.

2. Killian PJ, Hoffman GS (1980) Coexistence of systemic lupus erythematosus and myasthenia gravis. South Med J 73: 244-246.

3. Kister I, Gulati S, Boz C, Bergamaschi R, Piccolo G, et al. (2006) Neuromyelitis optica in patients with myasthenia gravis who underwent thymectomy. Arch Neurol 63: 851-856.

4. Marinó M, Ricciardi R, Pinchera A, Barbesino G, Manetti L, et al. (1997) Mild clinical expression of myasthenia gravis associated with autoimmune thyroid diseases. J Clin Endocrinol Metab 82: 438-443.

5. Peacey SR, Belchetz PE (1993) Graves' disease: associated ocular myasthenia gravis and a thymic cyst. J R Soc Med 86: 297-298.

6. Vakilian AR, Shafa MA, Kahnali JA, Rismanchian M (2006) Clinical manifestations, accompanying diseases, complications and thymus pathologies in 102 patients with myasthenia gravis. JRMS 11: 63-66

7. Kuks JB (2003) Clinical presentation and epidemiology of myasthenia gravis. In: Kaminski H, editor. Myasthenia Gravis and Related Disorders. First ed: Humana Press p. 93-114.

8. Oöpik M, Kaasik AE, Jakobsen J (2003) A population based epidemiological study on myasthenia gravis in Estonia. J Neurol Neurosurg Psychiatry 74: 1638-1643.

9. Osserman KE, Genkins G (1971) Studies in myasthenia gravis: review of a twenty-year experience in over 1200 patients. Mt Sinai J Med 38: 497-537.

10. Robertson NP, Deans J, Compston DA (1998) Myasthenia gravis: a population based epidemiological study in Cambridgeshire, England. J Neurol Neurosurg Psychiatry 65: 492-496.

11. Somnier FE, Keiding N, Paulson OB (1991) Epidemiology of myasthenia gravis in Denmark. A longitudinal and comprehensive population survey. Arch Neurol 48: 733-739.

12. Seybold ME (1999) Diagnosis of Myasthenia Gravis. In: Engel AG, editor. Myasthenia Gravis and myasthenic disorders. First ed: Oxford University Oress p. 146-66.
13. Lisak RP (1997) The clinical limits of myasthenia gravis and differential diagnosis. Neurology.48: S36-39.

14. Massey JM (1997) Acquired myasthenia gravis. Neurol Clin 15: 577-595.

15. Mayer SA (1997) Intensive care of the myasthenic patient. Neurology. 48: S70-75.

16. Bryan-Brown CW (1971) Contributions of the intensive care unit in the care of the myasthenia gravis patient. Mt Sinai J Med 38: 573-579.

17. Grob D (1999) Natural history of myasthenia gravis. In: Engel AG, editor. Myasthenia gravis and myasthenic disorders: Oxford University Pres p. 131-145.

18. Duyff RF, Van den Bosch J, Laman DM, van Loon BJ, Linssen WH (2000) Neuromuscular findings in thyroid dysfunction: a prospective clinical and electrodiagnostic study. J Neurol Neurosurg Psychiatry 68: 750-755.

19. Langouche L, Van den Berghe G (2006) The dynamic neuroendocrine response to critical illness. Endocrinol Metab Clin North Am 35: 777-791, ix.

20. Richman DP, Agius MA (2003) Treatment of autoimmune myasthenia gravis. Neurology 61: 1652-1661.

21. Kaplan I, Blakely BT, Pavlath GK, Travis M, Blau HM (1990) Steroids induce acetylcholine receptors on cultured human muscle: implications for myasthenia gravis. Proceedings of the National Academy of Sciences of the United States of America 87: 8100-8104.

22. Hollowell JG, Staehling NW, Flanders WD, Hannon WH, Gunter EW et al. (2002) Serum TSH, T(4), and thyroid antibodies in the United States population (1988 to 1994): National Health and Nutrition Examination Survey (NHANES III). J Clin Endocrinol Metab 87: 489-499.

23. PERRY SM (1950) Diabetes mellitus in association with myasthenia gravis. J Am Med Assoc 143: 1332-1333.

24. SCHANTZ ET (1952) Myasthenia gravis, diabetes mellitus and intraventricular block, as co-existent complications of pulmonary tuberculosis. Dis Chest 22: 183-186.

25. Jenkins EA, Hull RG, Gray RE, Hall MA, Ansell BM (1989) Diabetes mellitus and myasthenia gravis in a patient with systemic onset juvenile chronic arthritis. J R Soc Med 82: 368-369.

26. American Diabetes Association (2012) Standards of medical care in diabetes--2012. Diabetes Care 35 Suppl 1: S11-63.

27. Aguilar-Salinas CA, Velazquez Monroy O, Gomez-Perez FJ, Gonzalez Chavez A, Esqueda AL et al. (2003) Characteristics of patients with type 2 diabetes in Mexico: Results from a large population-based nationwide survey. Diabetes Care 26: 2021-2026.

28. Olaiz-Fernández G, Rivera-Dommarco J, Shamah-Levy T, Rojas R, Villalpando-Hernández S et al. (2006) Encuesta Nacional de Salud y Nutrición. instituto Nacional de Salud Pública.

29. Purvin V, Kawasaki A, Smith KH, Kesler A (2006) Statin-associated myasthenia gravis: report of 4 cases and review of the literature. Medicine (Baltimore) 85: 82-85.

30. Parmar B, Francis PJ, Ragge NK (2002) Statins, fibrates, and ocular myasthenia. Lancet 360: 717.

31. Hargreaves IP, Heales S (2002) Statins and myopathy. Lancet 359: 711-712. 